\title{
Bioeconomic Modelling: An Application to the North-East- Atlantic Cod Fishery
}

\author{
T. K. Kar (Corresponding author) \\ Department of Mathematics \\ Bengal Engineering and Science University, Shibpur \\ Howrah-711103, West Bengal, India \\ E-mail: t_k_kar@yahoo.com, tkar1117@gmail.com
}

Saroj Kumar Chattopadhyay

Department of Mathematics

Sree Chaityanya College, Habra, 24 PGs (North)

West Bengal, India

Research of T. K. Kar is financed by the Council of Scientific and Industrial Research (C S I R), India (Grant no. 25(0160)/ 08 / EMR-II dated 17.01.08)

\begin{abstract}
This paper describes a single species model, which followed by two stages, a mature and an immature stage. Conditions for existence of equilibrium points and their stability are discussed. The model in this paper has been developed on the concept of optimal management of resources based on the criterion of maximization of present values of net economic revenues. Using the data from the North-East Atlantic cod fishery, the results of the optimal stock, harvest and effort level are derived. Our simulation results show that optimal harvesting policy is much superior than the MSY policy and optimal paths always take less time than the suboptimal path to reach the optimal steady state. Our analysis also shows that, if it is insisted that a closure is take place for one of the two sub-stocks, it will be optimal to reduce fishing on the immature sub-stock rather than the mature sub-stock.
\end{abstract}

Keywords: Harvesting, Stage-structured, Global stability, MSY, Economic rent

\section{Introduction}

In the natural world, most of the species go through two or more life stages as they proceed from birth to death. Immature and mature are such two important stages that are to be taken into consideration. Most population models in the literature ignore such reality and assume that all individuals are identical and do not take into account any stage structure. It has been recognized that mortality, fertility and feeding depend on the size of the individuals. Some biological factors and some important rates (like rates of survival, reproduction) of individuals in a population almost always depend on stage-structure.

A stage-structured model of population growth consisting of immature and mature individuals was analyzed by Freedman and Gopalsamy (1986), where the stage-structured was modeled by introduction of a constant time delay. A prey-predator model with stage-structure for prey was established by Zhang et al. (2000) and obtained the necessary and sufficient condition of the permanence of the system. An overview on stage-structured models can be found in the recent book by Murdoch et al (2003). Recent papers of Kar (2003), Kar and Matsuda (2006), and Wiken (2004) study the stage-structure of species with or without time delays.

Harvesting has generally a strong impact on the population dynamics of a harvested species. The study of population dynamics with harvesting is a subject of mathematical bio-economics, and it is related to optimal management of renewable resources (Clark, 1990). Clark has also considered harvesting of a single species in a two fish ecologically competing population model. Chaudhuri $(1986,1988)$ studied combined harvesting and considered the perspectives of bio-economics 
and dynamic optimization of a two species fishery. Dai and Tang (1998) considered a prey-predator system with constant rate of harvesting. They showed how to approximate the region of asymptotic stability in biological terms in the initial states that ultimately lead to co-existence of the two species.

In recent years, the optimal management of renewable resources, which has direct relationship to sustainable development has been studied extensively by some authors. Generally, speaking, the exploitation of a species should be determined by the economic and biological values of the population. In this paper we aim to find an optimal harvesting policy which ensures a lasting exploitation of the biological resource and maximize the benefit resulting from the harvesting.

To develop a mathematical model for describing the interaction among immature and mature species we make the following assumptions:

(i) The recruitment of the mature species depends on the size of the immature species.

(ii) Two substocks interact via cannibalism.

(iii) Immature fish, which are product of mature fish, also become mature.

Cod fishery is a good example of the above model. An ecological interpretation of the cod cannibalism is that the cod has adopted to the cyclic recruitment pattern of capelin by eating its own offspring when the capelin stock is depleted and is in a state of rebuilding. It seems obvious if the nearly one and half a million tonnes of young cods eaten by older brothers and sisters in 1995-1997 had survived this could have prevented rebuilding of the capelin stocks in the subsequent years and thus threatened the food supply of the coming generations of cod. According to Eide (1993), following model is closely describing the North-East Atlantic cod stock.

In our model we consider the stage-structure of immature and mature of a single species. To establish a constructive management of commercial exploitation of biological resources we shall consider a special type linearly dependent harvesting efforts on both stages of the species. Let $x_{1}, x_{2}$ be the densities of immature, mature species respectively at any time $\mathrm{t}$. Then our proposed model is of the form

$$
\begin{aligned}
\frac{d x_{1}}{d t} & =r_{1} x_{1}\left(1-\frac{x_{1}}{a_{1} x_{2}}\right)-b_{1} x_{1} x_{2}, \\
\frac{d x_{2}}{d t} & =r_{2} x_{2}\left(1-\frac{x_{2}}{a_{2} x_{1}}\right),
\end{aligned}
$$

where $r_{1}, r_{2}, a_{1}, a_{2}$ and $b_{1}$ are all positive constants. Now considering the harvesting of both the species our model takes the form,

$$
\begin{aligned}
& \frac{d x_{1}}{d t}=r_{1} x_{1}\left(1-\frac{x_{1}}{a_{1} x_{2}}\right)-b_{1} x_{1} x_{2}-h_{1}, \\
& \frac{d x_{2}}{d t}=r_{2} x_{2}\left(1-\frac{x_{2}}{a_{2} x_{1}}\right)-h_{2} .
\end{aligned}
$$

We take the two forms of harvesting as follows:

$$
\begin{gathered}
h_{1}=q_{1}(1-\alpha) E x_{1}, h_{2}=q_{2} \alpha E x_{2}, \\
h_{1}=q_{1} E_{1} \alpha_{1} x_{1}, h_{2}=q_{2} E_{2} \alpha_{2} x_{2} .
\end{gathered}
$$

In (5), we consider the harvesting efforts $(1-\alpha) E$ and $\alpha E$ to the immature and mature species (where $0<\alpha<1)$ respectively i.e. some part of harvesting effort is applied for mature species and rest of the harvesting effort is for immature species and $q_{1}, q_{2}$ are respective catchability coefficient. In (6), the harvest $h_{i}=q_{i} E_{i} \alpha_{i} x_{i}$ where $q_{i}$ is the catchability coefficient, $E_{i}$ is the effort, and $\alpha_{i}$ is the fraction of the stock available to harvesting, with $0<\alpha_{i} \leq 1$.

\section{Results and discussion of the model taking $h_{i}(i=1,2)$ given in (5)}

\subsection{Equilibrium analysis}

We can analytically derive the equilibrium values of populations. Recalling equations (3) and (4), the equilibria are given by the intersection of the isoclines $\dot{x}_{1}=0=\dot{x}_{2}$.

It can be easily verified that the shape of the isocline $\dot{x}_{1}=0$ is a parabola but the $\dot{x}_{2}=0$ is a straight line and both depends on the effort $E$. The slope of the isocline $\dot{x}_{2}=0$ depends upon sign of the term $r_{2}-q_{2} \alpha E$. To allow the population to grow under such conditions, $r_{2}-q_{2} \alpha E$ is assumed to be positive, so that the isocline $\dot{x}_{2}=0$ has a positive slope. $r_{2}-q_{2} \alpha E>0$ 
implies that $\alpha E<r_{2} / q_{2}=B T P_{x_{2}}$ (biotechnical productivity of $x_{2}$ ). It follows from these considerations that there will be one strictly positive equilibrium $\left(x_{1}^{*}, x_{2}^{*}\right)$ at most, where

$$
\begin{gathered}
x_{1}^{*}=\frac{r_{2}}{a_{2}\left(r_{2}-q_{2} \alpha E\right)}\left[\frac{r_{1}\left\{a_{1} a_{2}\left(r_{2}-q_{2} \alpha E\right)-r_{2}\right\}}{b_{1} a_{1} a_{2}\left(r_{2}-q_{2} \alpha E\right)}-\frac{q_{1}(1-\alpha) E}{b_{1}}\right] \\
x_{2}^{*}=\frac{r_{1}\left[a_{1} a_{2}\left(r_{2}-q_{2} \alpha E\right)-r_{2}\right]}{b_{1} a_{1} a_{2}\left(r_{2}-q_{2} \alpha E\right)}-\frac{q_{1}(1-\alpha) E}{b_{1}}
\end{gathered}
$$

$<$ Table $1>$

$<$ Figure $1>$

$<$ Figure $2>$

Parameters $r_{i} \& k_{i}(i=1,2)$ and $b_{1}$ are taken from Eide (1993) and other parameters are taken from Armstrong and Sumalia (2000).

This equilibrium $\left(x_{1}^{*}, x_{2}^{*}\right)$ lies in $R_{+}^{2}$ if

$$
\frac{r_{1}\left[a_{1} a_{2}\left(r_{2}-q_{2} \alpha E\right)-r_{2}\right]}{b_{1} a_{1} a_{2}\left(r_{2}-q_{2} \alpha E\right)}>\frac{q_{1}(1-\alpha) E}{b_{1}}
$$

and

$$
E<r_{2} / q_{2} \alpha
$$

The condition (9) is equivalent to

$$
f(E)=q_{1} q_{2} \alpha(1-\alpha) E^{2}-\left[q_{1} r_{2}(1-\alpha)+r_{1} q_{2} \alpha\right] E+\left(r_{1} r_{2}-\frac{r_{1} r_{2}}{a_{1} a_{2}}\right)>0 .
$$

$<$ Figure $3>$

$<$ Figure $4>$

That is, $E$ lies outside the interval $[\lambda, \mu]$ where

$$
\lambda, \mu=\frac{\left[q_{1} r_{2}(1-\alpha)+q_{2} r_{1} \alpha\right] \mp \sqrt{\left[r_{2} q_{1}(1-\alpha)-r_{1} q_{2} \alpha\right]^{2}+4 r_{1} r_{2} q_{1} q_{2} \alpha(1-\alpha) / a_{1} a_{2}}}{2 q_{1} q_{2} \alpha(1-\alpha)}
$$

$\mu>0$ and we consider $a_{1} a_{2}>1$ to make $\lambda>0$. it is obvious that

$$
r_{2} / q_{2} \alpha<\mu
$$

and

$$
r_{2} / q_{2} \alpha>\lambda
$$

otherwise $\frac{4 r_{1} r_{2} q_{1} q_{2} \alpha(1-\alpha)}{a_{1} a_{2}}<0$

which is not true, because $r_{1}, r_{2}, q_{1}, q_{2}, a_{1}, a_{2}, \alpha$ are all positive and $\alpha<1$.

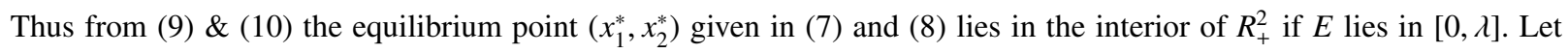
$\lambda=E_{0}$, then the sufficient condition that the system has a positive equilibrium is that

$$
E<E_{0}
$$

For $E=0$, the equilibrium $\left(x_{1}^{\prime}, x_{2}^{\prime}\right)$ is given by

$x_{1}^{\prime}=\frac{r_{1}\left(a_{1} a_{2}-1\right)}{b_{1} a_{1} a_{2}^{2}}, \quad x_{2}^{\prime}=\frac{r_{1}\left(a_{1} a_{2}-1\right)}{b_{1} a_{1} a_{2}}$ where $a_{1} a_{2}>1$.

The equilibrium curve for $E=0$ is then $x_{2}=a_{2} x_{1}$. Thus for $E=0$, the equilibrium curve originates from natural equilibrium $\left(x_{1}^{\prime}, x_{2}^{\prime}\right)$. As we increase harvesting effort the equilibrium point moves downwards and both the species become more extensively exploited. Both species will persist up to an effort level $E<E_{0}$. 


\subsection{Stability analysis}

We now inspect the local nature of equilibrium point $\left(x_{1}^{*}, x_{2}^{*}\right)$ in terms of stability. The Jacobian matrix of the system is

$$
J\left[x_{1}, x_{2}\right]=\left[\begin{array}{ll}
r_{1}\left(1-\frac{2 x_{1}}{a_{1} x_{2}}\right)-b_{1} x_{2}-q_{1}(1-\alpha) E & \frac{r_{1} x_{1}^{2}}{a_{1} x_{2}^{2}}-b_{1} x_{1} \\
\frac{r_{2} x_{2}^{2}}{a_{2} x_{1}^{2}} & r_{2}\left(1-\frac{2 x_{2}}{a_{2} x_{1}}\right)-q_{2} E \alpha
\end{array}\right]
$$

At $P\left(x_{1}^{*}, x_{2}^{*}\right)$ its characteristic equation is the quadratic in $\zeta$, given by

$$
\zeta^{2}+\left(\frac{r_{1} x_{1}^{*}}{a_{1} x_{2}^{*}}+\frac{r_{2} x_{2}^{*}}{a_{2} x_{1}^{*}}\right) \zeta+\frac{r_{2} b_{1}}{a_{2}} \frac{x_{2}^{* 2}}{x_{1}^{*}}=0
$$

By Hurwitz - Routh's condition the roots of this quadratic equation are real negative or complex with negative real parts if

$$
\left.\left(\frac{r_{1} x_{1}^{*}}{a_{1} x_{2}^{*}}+\frac{r_{2} x_{2}^{*}}{a_{2} x_{1}^{*}}\right)>0 \text { and } \frac{r_{2} b_{1}}{a_{2}} \frac{x_{2}^{* 2}}{x_{1}^{*}}>0\right\}
$$

These conditions are true if $P\left(x_{1}^{*}, x_{2}^{*}\right)$ exists in $R_{+}^{2}$. Thus the interior equilibrium $P\left(x_{1}^{*}, x_{2}^{*}\right)$ is locally stable if it exists. In otherwards we can say that the equilibrium remains stable up to a maximum effort level $E_{0}$ beyond which the system ceases to have a locally stable equilibrium.

$<$ Figure 5>

$<$ Figure 6>

To establish the non-existence of periodic orbit encircling $\left(x_{1}^{*}, x_{2}^{*}\right)$, we use Bendixon-Dulac criterion.

Let $\quad h\left(x_{1}, x_{2}\right)=1 / x_{1} x_{2}$. Obviously $h\left(x_{1}, x_{2}\right)>0$ for all $x_{1}, x_{2}>0$. We define

$$
\begin{gathered}
f_{1}\left(x_{1}, x_{2}\right)=r_{1} x_{1}\left(1-\frac{x_{1}}{a_{1} x_{2}}\right)-b x_{1} x_{2}-q_{1}(1-\alpha) E x_{1} \\
f_{2}\left(x_{1}, x_{2}\right)=r_{2} x_{2}\left(1-\frac{x_{2}}{a_{2} x_{1}}\right)-q_{2} \alpha E x_{2}
\end{gathered}
$$

Now we find that

$$
\frac{\partial}{\partial x_{1}}\left(f_{1} h\right)+\frac{\partial}{\partial x_{2}}\left(f_{2} h\right)=-\frac{r_{1}}{a_{1} x_{2}^{2}}-\frac{r_{2}}{a_{2} x_{1}^{2}}<0
$$

for all $x_{1}, x_{2}>0$, since all other parameters are strictly positive. Therefore, by Benedixon-Dulac criterion, there will be no periodic orbit within the interior of the first quadrant of state of space around $\left(x_{1}, x_{2}\right)$. So local stability of the equilibrium point $\left(x_{1}^{*}, x_{2}^{*}\right)$ implies the global asymptotic stability. From the point of view of ecological managers, it may be desirable to have an unique positive equilibrium which is globally asymptotic stable, in order to plan harvesting and keep sustainable development of ecosystem.

\subsection{Bionomic equilibrium}

The term bionomic equilibrium is the combined concept of biological equilibrium as well as economic equilibrium. For biological equilibrium $\dot{x}_{1}=0$ and $\dot{x}_{2}=0$, which implies, $x_{1}=0$ or, $E=\frac{r_{1}}{q_{1}(1-\alpha)}\left(1-\frac{x_{1}}{a_{1} x_{2}}\right)-\frac{b_{1}}{q_{1}(1-\alpha)} x_{2}$,

and $x_{2}=0$ or, $E=\frac{r_{2}}{q_{2} \alpha}\left(1-\frac{x_{2}}{a_{2} x_{1}}\right)$.

Therefore, for non-trivial biological equilibrium the solution lies on the curve,

$$
\left[\frac{r_{1}}{q_{1}(1-\alpha)}-\frac{r_{2}}{q_{2} \alpha}\right]-\frac{r_{1}}{q_{1}(1-\alpha)} \frac{x_{1}}{a_{1} x_{2}}+\frac{r_{2}}{q_{2} a_{2} \alpha} \frac{x_{2}}{x_{1}}-\frac{b_{1}}{q_{1}(1-\alpha)} x_{2}=0
$$

At an economic equilibrium the total revenue (TR) obtained by selling the harvested biomass is equal to the total cost (TC) for effort devoted to harvest. 
If $c_{1}=$ unit effort cost of immature species, $c_{2}=$ unit effort of mature species, $p_{1}=$ constant price per unit of biomass of immature species, $p_{2}=$ constant price per unit of biomass of mature species, then the economic rent i.e. net revenue (TR-TC) at any time $t$ is given by

$$
\pi\left(x_{1}, x_{2}, E\right)=\left[p_{1} q_{1}(1-\alpha) x_{1}+p_{2} q_{2} \alpha x_{2}-c\right] E
$$

Thus at economic equilibrium

$$
\left[p_{1} q_{1}(1-\alpha) x_{1}+p_{2} q_{2} \alpha x_{2}-c\right] E=0
$$

where $c=c_{1}(1-\alpha)+c_{2} \alpha$ and the bionomic equilibrium is the intersection of (18) and (19).

From (19) we get

$$
x_{1}=\frac{c-p_{2} q_{2} \alpha x_{2}}{p_{1} q_{1}(1-\alpha)}
$$

which exists in $R_{+}^{2}$ if

$$
x_{2}<c / p_{2} q_{2} \alpha
$$

At the intersection of (20) and (18)

$$
\left[\frac{r_{1}}{q_{1}(1-\alpha)}-\frac{r_{2}}{q_{2} \alpha}\right]-\frac{r_{1}}{q_{1}(1-\alpha) a_{1}} \frac{c-p_{2} q_{2} \alpha x_{2}}{p_{1} q_{1} x_{2}(1-\alpha)}+\frac{r_{2}}{q_{2} a_{2} \alpha} \frac{x_{2} p_{1} q_{1}(1-\alpha)}{c-p_{2} q_{2} \alpha x_{2}}-\frac{b_{1} x_{2}}{q_{1}(1-\alpha)}=0
$$

Simplifying this we get the cubic equation in $x_{2}$,

$$
A x_{2}^{3}+B x_{2}^{2}+C x_{2}+D=0
$$

where

$$
\begin{gathered}
A=a_{1} a_{2} A_{3} A_{4} A_{5} \\
B=-\left[a_{1} a_{2} A_{3}\left(A_{1}-A_{2}\right) A_{4}+A_{1} A_{4}^{2} a_{2}-a_{1} A_{2} A_{3}^{2}+a_{1} a_{2} A_{3} A_{5} c\right] \\
C=a_{1} a_{2} A_{3}\left(A_{1}-A_{2}\right) c+2 A_{1} A_{4} a_{2} c \\
D=-A_{1} a_{2} c
\end{gathered}
$$

and $A_{1}=\frac{r_{1}}{q_{1}(1-\alpha)}, A_{2}=\frac{r_{2}}{q_{2} \alpha}, A_{3}=p_{1} q_{1}(1-\alpha), A_{4}=p_{2} q_{2} \alpha, A_{5}=\frac{b_{1}}{q_{1}(1-\alpha)}$.

Since $a_{1}, a_{2}, q_{1}, q_{2}, r_{1}, r_{2}, \alpha, p_{1}, p_{2}, c$ are all positive we have $A>0$ and $D<0$. The co-efficient $\mathrm{B}$ and $\mathrm{C}$ may be positive, negative or zero. So the following cases may appear:

Case I. When both $B$ and $C$ are positive then all the co-efficients of (23) are positive except the last co-efficient $D$. Hence by Descarte's rule of signs (23) has exactly one positive root. That is in this case unique positive bionomic equilibrium exists if (21) is satisfied.

Case II. When both $B$ and $C$ are negative then all the co-efficients of (23) are negative except the leading coefficient which is essentially positive. Hence (23) has only one positive root and so unique interior bionomic equilibrium exists if (21) is satisfied.

Case III. When $B>0$ and $C<0$, first two co-efficients of (23) are positive and last two are negative. Hence by Descarte's rule of signs (23) has exactly one positive root that is in turn unique positive bionomic equilibrium exists if (21) is true.

$<$ Figure $7>$

$<$ Figure $8>$

Case IV. When $B<0, C>0$ then equation (23) may have more than one positive roots and system may have non-unique interior bionomic equilibrium if (21) is also true.

Case V. In all the cases when $B=0 \&$ either $C>0$ or $<0$ and $C=0, B>0$ or $<0$ and $B=0, C=0$ the equation (23) has exactly one positive root i.e. the system has unique interior bionomic equilibrium if (21) is also true. 
From all the cases studied above it is clear that equation (23) has at least one positive root. If this positive root be less than $c / p_{2} q_{2} \alpha$ we denote it by $x_{2 \infty}$ and from (20) we get a value of $x_{1}$ denoted by $x_{1 \infty}$ and the bionomic equilibrium point $R\left(x_{1 \infty}, x_{2 \infty}\right)$ is obtained. Now (23) will have a positive root less than $c / p_{2} q_{2} \alpha$ i.e. a root in $\left(0, c / p_{2} q_{2} \alpha\right)$ if $\left(A x_{2}^{3}+B x_{2}^{2}+C x_{2}+D\right)$ has opposite signs for $x_{2}=0$ and $x_{2}=c / p_{2} q_{2} \alpha$ that is if

$\left(a_{1} a_{2} p_{1} p_{2} q_{2} b_{1} \alpha\right)\left(c / p_{2} q_{2} \alpha\right)^{3}$

$$
\begin{gathered}
-\left[a_{1} a_{2} p_{1} p_{2}\left\{r_{1} q_{2} \alpha-r_{2} q_{1}(1-\alpha)\right\}+\frac{r_{1} a_{2} p_{2}^{2} q_{2}^{2} \alpha^{2}}{q_{1}}-\frac{r_{2}}{q_{2} \alpha} a_{1} p_{1}^{2} q_{1}^{2}(1-\alpha)^{2}+a_{1} a_{2} p_{1} b_{1} c\right] \\
\left(\frac{c}{p_{2} q_{2} \alpha}\right)^{2}+\left[\frac{a_{1} a_{2} p_{1} c}{q_{2} \alpha}\left\{r_{1} q_{2} \alpha-r_{2} q_{1}(1-\alpha)\right\}+\frac{2 r_{1} a_{2} p_{2} q_{2} \alpha c}{q_{1}(1-\alpha)}\left(\frac{c}{p_{2} q_{2} \alpha}\right)-\frac{a_{2} r_{1} c}{q_{1}(1-\alpha)}\right]>0 .
\end{gathered}
$$

Thus the system has a bionomic equilibrium if (24) is satisfied by the parameters of the model.

Then for $40 \%$ and $60 \%$ of the effort level devoted to mature species i.e. for $\alpha=0.4$ and $\alpha=0.6$ the inequation (23) is satisfied. For $\alpha=0.4$ the bionomic equilibrium point is $(0.33,0.37)$ and for $\alpha=0.6$ the bionomic equilibrium is attained at the point $(0.31,0.32)$.

\subsection{Optimal harvest policy}

In this section we shall discuss the optimal harvest policy. For that we consider the present value $J$ of a continuous time-stream of revenues as

$$
J=\int_{0}^{\infty} e^{-\delta t}\left[p_{1} q_{1}(1-\alpha) x_{1}+p_{2} q_{2} \alpha x_{2}-c\right] E d t
$$

where, $\delta$ denotes the instantaneous annual rate of discount. Our problem is to maximize $J$ subject to the state equation (3) and (4) by invoking Pontryagin et al. (1962). The control variable $E(t)$ is subjected to the constraint set $0 \leq E \leq E_{\max }$. We consider the current value of Hamiltonian as

$$
H=\left[p_{1} q_{1}(1-\alpha) x_{1}+p_{2} q_{2} \alpha x_{2}-c\right] E+\mu_{1}\left[x_{1}\left\{r_{1}\left(1-\frac{x_{1}}{a_{1} x_{2}}\right)-b_{1} x_{2}-q_{1}(1-\alpha) E\right\}\right]+\mu_{2}\left[x_{2}\left\{r_{2}\left(1-\frac{x_{2}}{a_{2} x_{1}}\right)-q_{2} \alpha E\right\}\right]
$$

where $\mu_{1}(t), \mu_{2}(t)$ are adjoint variables. The adjoint equations are

$$
\begin{aligned}
& \frac{d \mu_{1}}{d t}=\delta \mu_{1}-\frac{\partial H}{\partial x_{1}} \\
& \frac{d \mu_{2}}{d t}=\delta \mu_{2}-\frac{\partial H}{\partial x_{2}} .
\end{aligned}
$$

Here we use the steady state solution as we are concerned with optimal equilibrium and we consider $x_{1}, x_{2}$ as constants in the subsequent steps.

Then using the steady state solution the adjoint equations (27) become,

$$
\begin{gathered}
\frac{d \mu_{1}}{d t}=\left(\delta+\frac{r_{1} x_{1}}{a_{1} x_{2}}\right) \mu_{1}-\mu_{2} \frac{r_{2} x_{2}^{2}}{a_{2} x_{1}^{2}}-p_{1} q_{1}(1-\alpha) E, \\
\frac{d \mu_{2}}{d t}=\left(\delta+\frac{r_{2} x_{2}}{a_{2} x_{1}}\right) \mu_{2}-\mu_{1}\left(\frac{r_{1} x_{1}^{2}}{a_{1} x_{2}^{2}}-b_{1} x_{1}\right)-p_{2} q_{2} \alpha E .
\end{gathered}
$$

We write these equations as

$$
\begin{aligned}
& \left(D-m_{1}\right) \mu_{1}+m_{2} \mu_{2}=-m_{3} \\
& \left(D-m_{4}\right) \mu_{2}+m_{5} \mu_{1}=-m_{6}
\end{aligned}
$$

where $D \equiv d / d t$, and

$$
m_{1}=\delta+\frac{r_{1} x_{1}}{a_{1} x_{2}}, \quad m_{2}=\frac{r_{2} x_{2}^{2}}{a_{2} x_{1}^{2}}, \quad m_{3}=p_{1} q_{1}(1-\alpha) E,
$$




$$
m_{4}=\delta+\frac{r_{2} x_{2}}{a_{2} x_{1}}, \quad m_{5}=\frac{r_{1} x_{1}^{2}}{a_{1} x_{2}^{2}}-b_{1} x_{1}, \quad m_{6}=p_{2} q_{2} \alpha E .
$$

Eliminating $\mu_{1}$ from the equation (29) we get the differential equation

$$
\begin{gathered}
{\left[D^{2}-\left(m_{1}+m_{4}\right) D+m_{1} m_{4}-m_{2} m_{5}\right] \mu_{2}=m_{1} m_{6}+m_{3} m_{5}} \\
\therefore \mu_{2}=A e^{\lambda_{1} t}+B e^{\lambda_{2} t}+\frac{m_{1} m_{6}+m_{3} m_{5}}{m_{1} m_{4}-m_{2} m_{5}}
\end{gathered}
$$

where $\mathrm{A}, \mathrm{B}$ are arbitrary constants and $\lambda_{1}, \lambda_{2}$ are the roots of

$$
\lambda^{2}-\left(m_{1}+m_{4}\right) \lambda+m_{1} m_{4}-m_{2} m_{5}=0
$$

It is clear that $\mu$ is bounded if $\mathrm{A}, \mathrm{B}$, are zero or $\lambda_{1}, \lambda_{2}<0$. We consider, for simplicity, that A,B are zero.

$$
\therefore \mu_{2}=\frac{m_{1} m_{6}+m_{3} m_{5}}{m_{1} m_{4}-m_{2} m_{5}}
$$

. Similarly we can derive that $\mu_{1}=\frac{m_{3} m_{4}+m_{2} m_{6}}{m_{1} m_{4}-m_{2} m_{5}}$.

Now $\partial H / \partial E=0$ implies

$$
p_{1} q_{1}(1-\alpha) x_{1}+p_{2} q_{2} \alpha x_{2}-c=\mu_{1} q_{1}(1-\alpha) x_{1}+\mu_{2} q_{2} \alpha x_{2} .
$$

Using the values of $\mu_{1}, \mu_{2}$ we get,

$$
\begin{aligned}
\left(p_{1} q_{1}(1-\alpha) x_{1}+p_{2} q_{2} \alpha x_{2}-c\right)\left(\left(\delta+\frac{r_{1} x_{1}}{a_{1} x_{2}}\right)\left(\delta+\frac{r_{2} x_{2}}{a_{2} x_{1}}\right)-\frac{r_{2} x_{2}^{2}}{a_{2} x_{1}^{2}}\left(\frac{r_{1} x_{1}^{2}}{a_{1} x_{2}^{2}}-b_{1} x_{1}\right)\right) \\
=\left[\left(\delta+\frac{r_{2} x_{2}}{a_{2} x_{1}}\right) p_{1} q_{1}(1-\alpha)+\frac{r_{2} x_{2}^{2}}{a_{2} x_{1}^{2}} p_{2} q_{2} \alpha\right] E q_{1}(1-\alpha) x_{1} \\
+\left[\left(\delta+\frac{r_{1} x_{1}}{a_{1} x_{2}}\right) p_{2} q_{2} \alpha+p q(1-\alpha)\left(\frac{r_{1} x_{1}^{2}}{a_{2} x_{2}^{2}}-b_{1} x_{1}\right)\right] E q_{2} \alpha x_{2} .
\end{aligned}
$$

Solving (31), (10) \& (11) we can get a value of E, the optimal harvesting effort.

Since the optimal control problem is linear of its control variable E, the optimal harvesting policy will be a combination of bang-bang and singular controls. Let $\mathrm{T}$ be the time taken to reach the optimal state by using the bang-bang control. Then the optimal control policy will be

$$
E(t)=\left\{\begin{array}{l}
\tilde{E} \text { for } 0 \leq t \leq T \\
E^{*} \text { for } t>T
\end{array}\right.
$$

where

$$
\bar{E}(t)=\left\{\begin{array}{l}
E_{\text {max }} \text { for } \sigma(t)>0 \\
E_{\text {max }} \text { for } \sigma(t)<0,
\end{array}\right.
$$

here

$$
\sigma(t)=p_{1} q_{1}(1-\alpha) x_{1}+p_{2} q_{2} \alpha x_{2}-c-\mu_{1} q_{1}(1-\alpha) x_{1}-\mu_{2} q_{2} \alpha x_{2}
$$

is the switching function.

$<$ Figure 9-11>

Phase diagram showing the changes in the equilibrium biomass as a response to changes in the effort allocation. Both the equilibrium biomass tends to zero with the efforts increases. Here $\alpha=0.4$ for (a) and $\alpha=0.6$ for (b).

$<$ Figure $12><$ Figure 13> 
Then the following table shows the interior equilibrium $\left(x_{1}^{*}, x_{2}^{*}\right) \&$ optimal harvest $E^{*}, E_{\max }, E_{\min }$ for $40 \%$ and $60 \%$ of the effort devoted to the mature species.

$<$ Table 2>

The following table shows the time taken by different approach paths to reach an equilibrium point.

$<$ Table 3>

Management of renewable resources has generally been relied on the concept of Maxium Sustainable Yield (MSY). Recently, several objections have been raised against the use of MSY on both biological and socioeconomic grounds (Matsuda and Abrams, 2006). One of the most serious objection is obviously the non-recognition of the cost factor. The inadequacy of MSY concept has resulted in a trend to replace it with a concept of optimal resource management based on criterion of maximization of present values of net economic revenues. In this paper we have attempted to find optimal steady state solution (i.e. optimal stock, optimal harvest and effort level) that ensures the long run sustainability of the resource and excludes the possibility of depletion due to over exploitation and give maximum benefit.

Approach paths of optimal and suboptimal harvesting policy are depicted in figures 12 and 13. Optimal paths always take less time than the suboptimal path to reach the optimal steady state.

In both the cases for $\alpha=0.4$ and $\alpha=0.6$, it is observed that optimal harvesting effort $E^{*}<E_{M S Y}<E_{\text {max }}$. The MSY are 0.084 (for $\alpha=0.4$ ) and 0.16 (for $\alpha=0.6$ ) and the effort for producing the said amounts are 95.1 units and 129.6 units respectively. Also for the optimal harvesting effort $E^{*}$, the optimal steady state is globally asymptotically stable. Optimal catch are $(0.2136+0.0722)=0.2858$ (for $\alpha=0.4)$ and $(0.2236+0.0649)=0.2885$ (for $\alpha=0.6$ ). Optimal efforts for producing the said optimal catch are 48.06 and 74.511 respectively. It clearly implies that the higher level of effort causes overfishing which, in turn, causes lower stock. As a consequence, even higher level of effort in later years does not get adequate quantity of catch.

\section{Results and discussion of the model using catch functions}

$$
h_{i}=q_{i} E_{i} \alpha_{i} x_{i}, i=1,2 \text { given in (6) }
$$

In recent years, serious stock decline or collapse is a major risk for most of the world fisheries. Marine reserve may be a possible way to reduce this risk by increasing the equilibrium stock size and other effects such as preserving spawning and nursery growths. It may influence biomass growth in various ways. It is for instance possible that marine reserves, by providing some of the fish with a sanctuary from distributing fishing activity may actually enhance biomass growth. The same effect may occur if marine reserves manage to protect fast growing immature fish or conserve habitat variables important for fish survival and growth.

The management goal is overall profit maximization with the profit function

$$
\pi=p_{1} q_{1} E_{1} \alpha_{1} x_{1}+p_{2} q_{2} E_{2} \alpha_{2} x_{2}-c_{1} E_{1}-c_{2} E_{2} .
$$

The mathematical formulation is given by

$$
\text { maximize } \int_{0}^{\infty} \pi e^{-\delta t} d t
$$

subject to

$$
\begin{gathered}
\frac{d x_{1}}{d t}=r_{1} x_{1}\left(1-\frac{x_{1}}{a_{1} x_{2}}\right)-b_{1} x_{1}-q_{1} E_{1} \alpha_{1} x_{1} \\
\frac{d x_{2}}{d t}=r_{2} x_{2}\left(1-\frac{x_{2}}{a_{2} x_{1}}\right)-q_{2} E_{2} \alpha_{2} x_{2}, \\
0 \leq E_{i} \leq E_{\text {imax }} .
\end{gathered}
$$

We have applied the maximum principle to the optimal control problem (as in section 2) and the parameter values of Table 1.

Equilibrium profits for different closure alternatives are given below.

$<$ Table 4-8>

It is already established that marine reserves are a practical means of managing resource populations and are therefore, beneficial for conserving the ecological environment and resource populations (Kar \& Matsuda, 2008). Using the data 
from North-East Atlantic cod fishery, our analysis shows that, if it is insisted that a closure is to take place for one of the two sub-stocks, it will be optimal to reduce fishing on the immature sub-stock rather than the mature sub-stock. This supports the case for greater focus on regulation of harvest upon juvenile fish, as may be claimed to be the case in most fisheries in recent times. For the cod fishery, there is very little difference in resulting profits from a fishery with no reserves and a fishery where half of the immature fish stock is projected.

Introduction of marine reserves uniformly increases the optimal equilibrium and reduce the profitability of harvesting for a given biomass. Both effects serve to increase the equilibrium biomass level. Due to these opposing forces, the impact of marine reserves on optimal fishing effort level is indeterminate. Thus for the cod fishery, introduction of marine reserves is more likely to reduce net benefits from the fishery. Thus, inspite of a certain increase in stock biomass, introducing marine reserves in the cod fishery seems rather not attractive in general.

\section{Conclusions}

Past experience has shown that over fishing may cause extinction or near extinction of different species of fishes, like Antarctic blue whales, Peruvian anchoveta and Canadian cod fishery etc. North-East Atlantic cod stocks have suffered from both over fishing and food shortages, as stocks of their prey species herring and capelin have fluctuated. The whole stock is so unstable that the threat of a collapse is imminent unless fisheries scientists recommendations are followed. According to ICES, the current fishing levels are unstable. ICES has consequently called for a recovery plan to protect these stocks. In 2003, ICES stated that there is a high risk of stock collapse if current exploitation levels continue and recommended a zero catch of Atlantic cod in the year 2004. In the year 2004, estimated cod stock size was 1.6 million tones. Experts from the International Council for the Exploitation of Seas (ICES) have proposed that cod catches should be limited to $50 \%$ of catch levels in 2006 to enable young fish to mature and build up the population.

We assume that one of the objectives of the fishery is to find an optimal harvesting policy which ensures a lasting exploitation of the resource and other is to maximize the benefit resulting from the harvesting.

If we analyze our results and compare these with the real situation that exists in the last year of the period, it is clear that the North-East Atlantic cod fishery is being exploited in an inefficient way, from an economic view point as well as with the conservation of the resource. By comparing optimal stock and harvest with its actual stock and harvest, this study indicates the fact that both stocks and harvest at the current level is much lower than its optimal level. Over fishing during the earlier period may have had some consequences on population dynamics of the species. The biomass level in 2004 was significantly lower than the optimal equilibrium level. Therefore in order to achieve optimal steady solution, it is necessary to proper regulation of the fishery and reduction of fishing pressure on it.

The results of the study presented in this paper conclude that (i) North-East Atlantic cod fishery is not managed and utilized optimally, (ii) present condition of less biomass stock indicates that the danger of depletion of the resource can not be ruled out, (iii) if corrective measures are taken, NEAC stock would take less time to reach its optimal steady state, (iv) The quickest possible means of reaching an efficient solution is by means of so-called bang-bang controls, i.e., not exercising fishing effort in the fishery until the resource recovers its optimum levels, (v) Herring and capelin are the most important prey for Atlantic cod, so the survival of their stocks also vital for cod. Thus the interaction between cod and its preys like capelin and herring are of fundamental importance to the dynamic of the processes which govern the fish production in the region.

In the present model many important variables such as the environmental effects and interaction with other species are disregarded. Hence, the results obtained in this study should be taken with care.

\section{References}

Armstrong, C. W \& Sumaila U. R. (2000). Cannibalism and the optimal sharing of the North-East Atlantic cod stock: A bioeconomic model, J. Bioecon, 2, 99-115.

Chaudhuri, K. S. (1986). A bio-economic model of harvesting: a multi species fishery. Ecol. Model, 32, $267-279$.

Chaudhuri, K. S. (1988). Dynamic optimization of combined harvestings of a two species fishery, Ecol. Model, 32, 267279.

Clark, C. W. (1990). Mathematical Biosciences: The optimal Management of Renewable Resources: Wily, New York.

Dai, G. \& Tang, M. (1998). Consistence region and global dynamics of a harvested predator-prey system. Siam J. Appl. Math, 58,193-210.

Eide, A. (1993). Fluctuations in the cod stock (in Norwegion). Mimeo, Norwegion College of Fishery Science, University of Troms, Troms, Norway.

Freedman, H. I., \& Gopalsamy, K. (1986). Global stability in time delayed single species dynamics. Bulletin of Mathematical Biology, 48(5/6), 485 -492.

Kar, T. K. (2003). Selective harvesting in a prey predator fishing with time delay. Math. Comp. Model, 38, $449-458$. 
Kar, T. K. and Matsuda H. (2006). Controllability of a harvested prey-predator system with time delay. J. Biol. Syst. 14, 1-12.

Kar T. K. and Matsuda H. (2008). A bioeconomic model of a single species fishery with marine reserve, 86, 171-180.

Matsuda, H and Abrams P. (2006). Maximal yields from multispecies fisheries systems: Rules for systems with multiple tropic levels. Ecol. Appl. 16, 225-237.

Murdoch, W. W, Briggs, C. J., \& Nisbet R. M. (2003). Consumer Resource Dynamics, Princeton Univ. Press, Princeton.

Pontryagin, L. S., Boltyonsku, V. G., Gamkrelidre, R. V. and Mishchenko, E. F. (1962). The Mathematical Theory of Optimal Process, Wiley, New York.

Wikan, A. (2004). Dynamical consequences of harveste in discrete age-structured of population models. J. Math. Biol. 49, 35-55.

Zhang, X., Chen, L. \& Neumann U. A. (2000). The stage-structured predator-prey model and optimal harvesting policy. Mathematical Biosciences, 168, 201-210.

Table 1. Estimated values of the parameters

\begin{tabular}{|c|c|c|c|c|c|c|c|c|c|}
\hline Parameters & $r_{1}$ & $r_{2}$ & $a_{1}$ & $a_{2}$ & $b_{1}$ & $q_{1}$ & $q_{2}$ & $p_{1}$ & $p_{2}$ \\
\hline Values & 0.5003 & 0.6728 & 8.7608 & 1.1880 & 0.2023 & 0.006650 & 0.001175 & 7579 & 8655 \\
\hline Parameters & $c_{1}$ & $c_{2}$ & $\bar{\delta}$ & & & & & & \\
\hline Values & 18.602103 & 1.452341 & 0.01 & & & & & & \\
\hline
\end{tabular}

Table 2.

\begin{tabular}{|c|c|c|c|c|c|}
\hline$\alpha$ & $x_{1}^{*}$ & $x_{2}^{*}$ & $E^{*}$ & $E_{\max }$ & $E_{\min }$ \\
\hline 0.4 & 1.113845 & 1.279392 & 48.06224 & 112.3152 & 0 \\
\hline 0.6 & 1.128144 & 1.235587 & 74.51134 & 166.2006 & 0 \\
\hline
\end{tabular}

Table 3.

\begin{tabular}{|c|c|c|c|c|}
\hline Figure no. & \multicolumn{2}{|c|}{ Co-ordinates of } & Path type & $\begin{array}{c}\text { Time taken to reach } \\
\text { the end point }\end{array}$ \\
\cline { 2 - 3 } & Initial point & End point & & 11.67 \\
\hline$(\alpha=0.4)$ & $(0.2,0.3)$ & $(1.114,1.278)$ & Optimal & 20 \\
Figure 12 & & $(1.1150,1.2791)$ & Suboptimal & 10.83 \\
\hline$(\alpha=0.6)$ & $(0.2,0.3)$ & $(1.129,1.255)$ & Optimal & 20.8 \\
Figure 13 & & $(1.1346,1.236)$ & Suboptimal & 20.8 \\
\hline
\end{tabular}

Table 4.

\begin{tabular}{|l|c|c|}
\hline & $\alpha_{1}=1, \alpha_{2}=1$ & $\alpha_{1}=0.5, \alpha_{2}=0.5$ \\
\hline Profit & 6660.24 & 5938.74 \\
\hline $\begin{array}{l}\text { Optimal equi- } \\
\text { librium }\end{array}$ & $(3.71,1.38)$ & $(3.85,1.47)$ \\
\hline
\end{tabular}

Table 5. $\alpha_{1}=1$

\begin{tabular}{|l|c|c|c|c|}
\hline$\alpha_{2}$ & 0.3 & 0.7 & 0.8 & 0.9 \\
\hline Profit & 5343.18 & 6415.10 & 6517.08 & 6596.56 \\
\hline $\begin{array}{l}\text { Optimal equi- } \\
\text { librium }\end{array}$ & $(3.58,1.43)$ & $(3.69,1.39)$ & $(3.7,1.38)$ & $(3.7,1.38)$ \\
\hline
\end{tabular}

Table 6. $\alpha_{2}=1$

\begin{tabular}{|l|c|c|c|c|c|}
\hline$\alpha_{1}$ & 0.3 & 0.4 & 0.5 & 0.6 & 0.9 \\
\hline Profit & 6451.15 & 6447.46 & 6509.1 & 6555.04 & 6641.52 \\
\hline $\begin{array}{l}\text { Optimal equi- } \\
\text { librium }\end{array}$ & $(4.15,1.54)$ & $(4.00,1.48)$ & $(3.91,1.45)$ & $(3.84,1.42)$ & $(3.73,1.38)$ \\
\hline
\end{tabular}


Table 7. $\alpha_{1}=0.5$

\begin{tabular}{|l|c|c|c|c|c|c|c|}
\hline$\alpha_{2}$ & 0.1 & 0.3 & 0.4 & 0.5 & 0.6 & 0.9 & 1 \\
\hline Profit & 1790.16 & 5191.5 & 5656.73 & 5938.59 & 6127.92 & 6445.09 & 6508.81 \\
\hline $\begin{array}{l}\text { Optimal } \\
\text { equilibrium }\end{array}$ & $(3.08,1.74)$ & $(3.77,1.51)$ & $(3.82,1.48)$ & $(3.85,1.47)$ & $(3.87,1.46)$ & $(3.90,1.45)$ & $(3.91,1.45)$ \\
\hline
\end{tabular}

Table 8. $\alpha_{2}=0.5$

\begin{tabular}{|l|c|c|c|c|c|c|c|}
\hline$\alpha_{1}$ & 0.3 & 0.4 & 0.5 & 0.6 & 0.7 & 0.8 & 0.9 \\
\hline Profit & 5798.16 & 4901.05 & 5938.77 & 5984.93 & 6020.59 & 6048.8 & 6071.6 \\
\hline $\begin{array}{l}\text { Optimal equi- } \\
\text { librium }\end{array}$ & $(4.1,1.57)$ & $(3.95,1.51)$ & $(3.85,1.47)$ & $(3.79,1.45)$ & $(3.74,1.45)$ & $(3.71,1.42)$ & $(3.68,1.41)$ \\
\hline
\end{tabular}

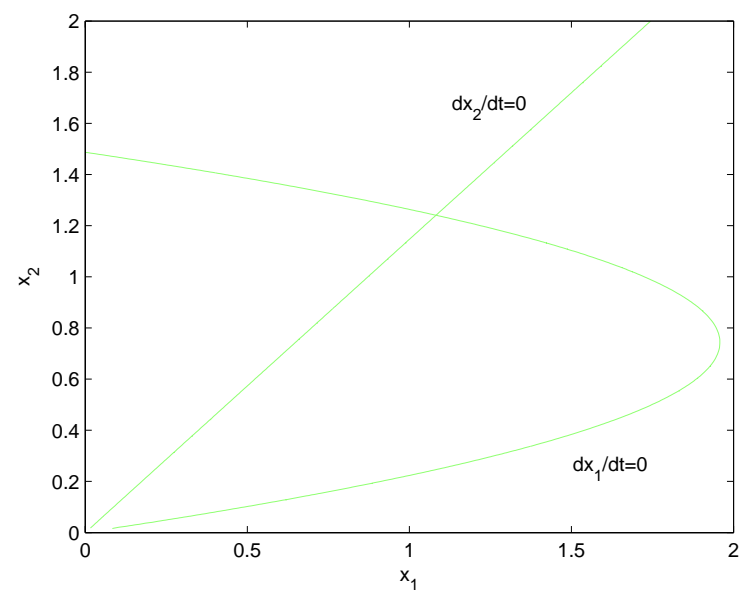

Figure 1. Isoclines $\dot{x}_{1}=0$ and $\dot{x}_{2}=0$ for $\alpha=0.4$

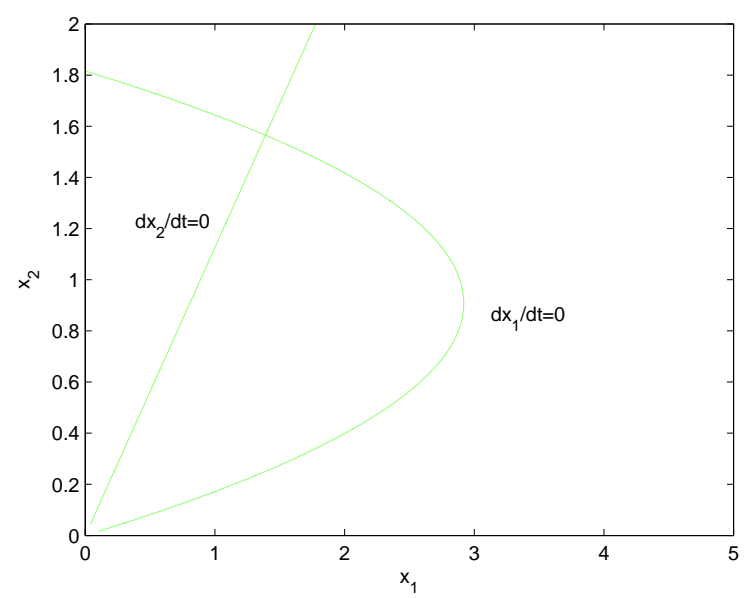

Figure 2. Isoclines $\dot{x}_{1}=0$ and $\dot{x}_{2}=0$ for $\alpha=0.6$ 


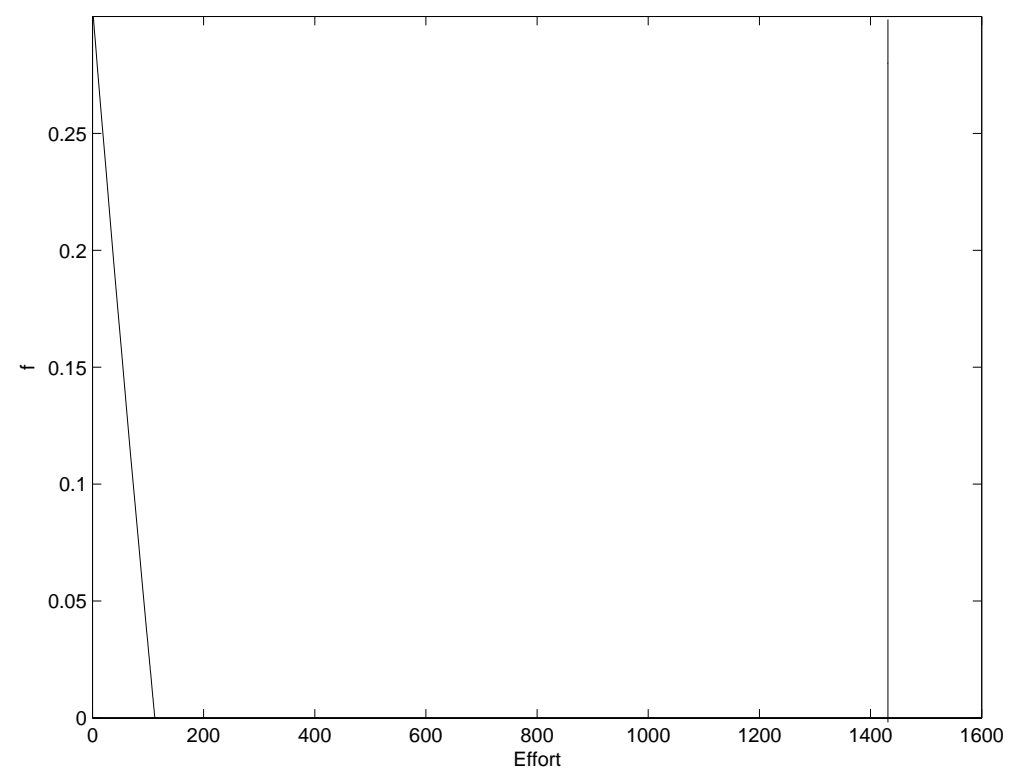

Figure 3. This figure shows that $f(E)>0$ for $E<112.3$ and so it make some sense (here $\alpha=0.4$.)

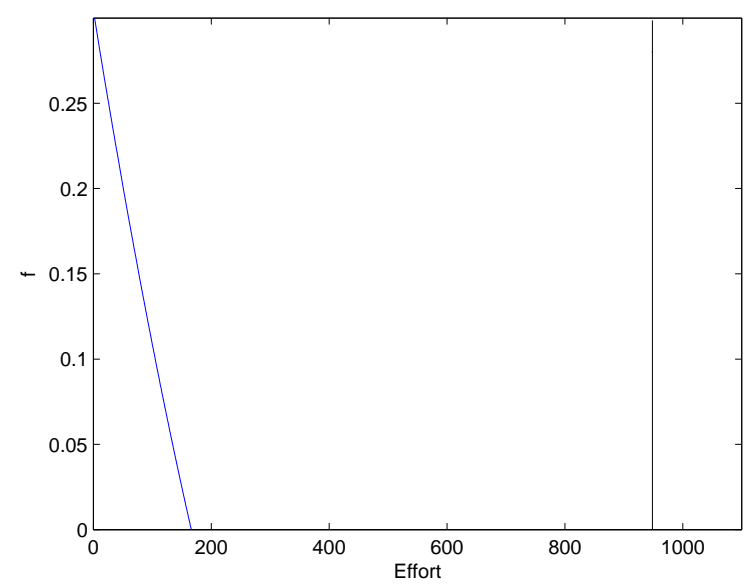

Figure 4. This figure shows that $f(E)>0$ for $E<166.2$ and so it make some sense (here $\alpha=0.6$ )

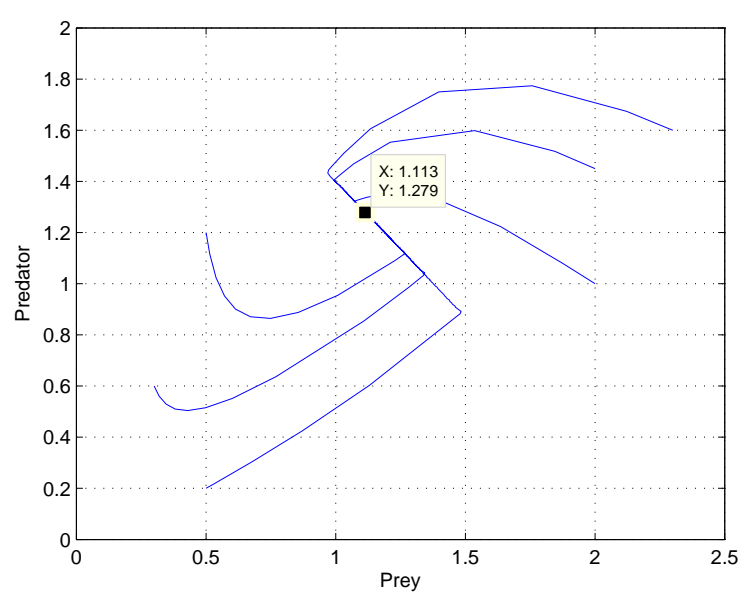

Figure 5. Phase plane trajectories with $\alpha=0.4$. 


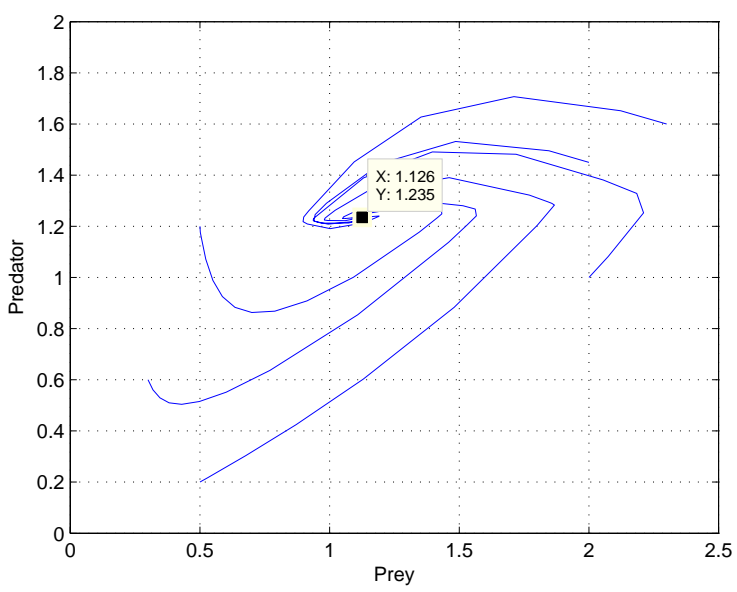

Figure 6. Phase plane trajectories with $\alpha=0.6$.

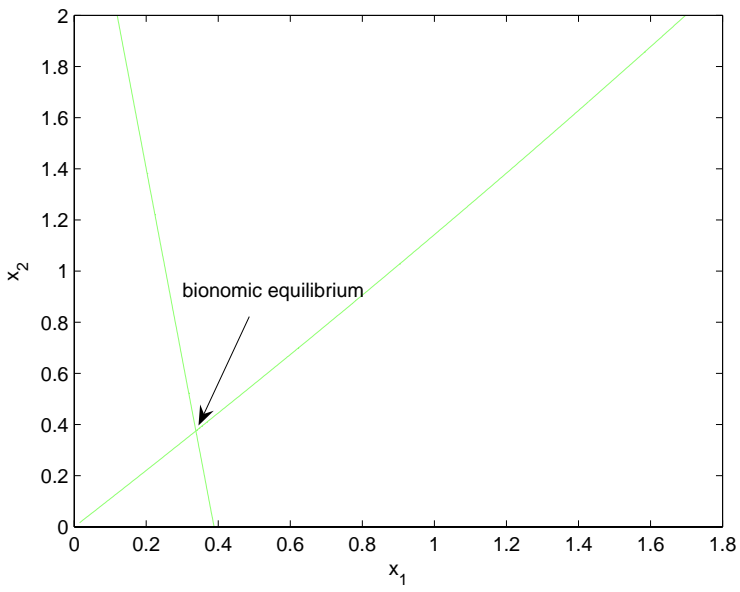

Figure 7. Phase diagram showing the unique bionomic equilibrium exists for $\alpha=0.4$.

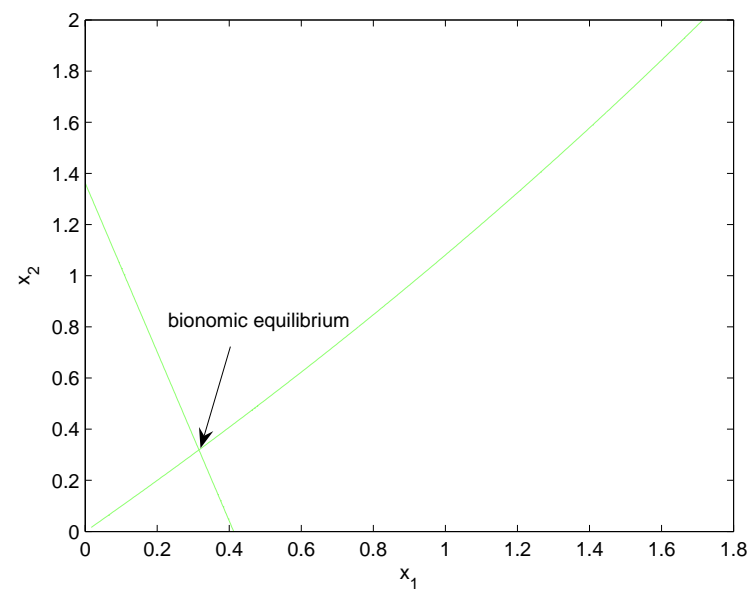

Figure 8. Phase diagram showing the unique bionomic equilibrium exists for $\alpha=0.6$. 


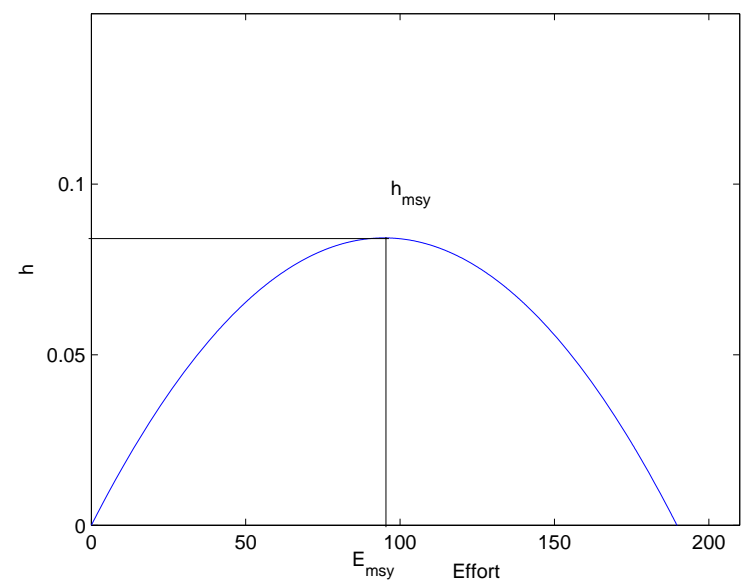

Figure 9. The Maximum Sustainable Yield (MSY) for $\alpha=0.4$. Here $E_{M S Y}=95.10$ units and $h_{M S Y}=0.084$.

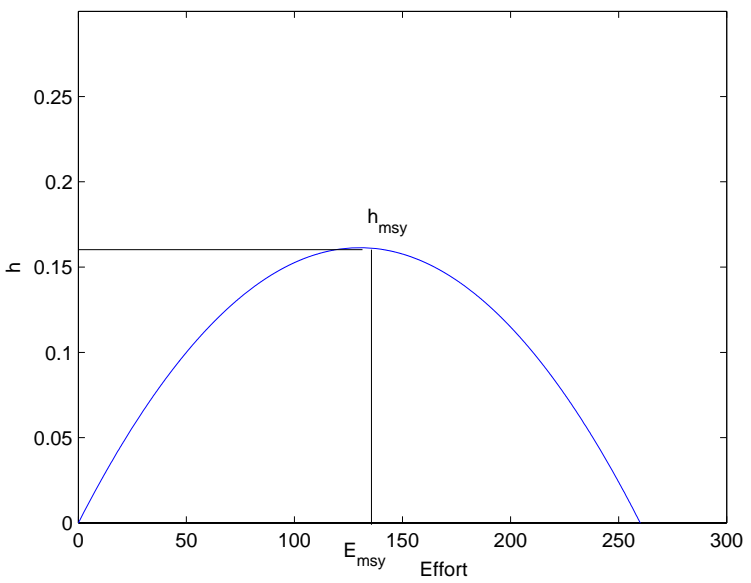

Figure 10. The Maximum Sustainable Yield (MSY) for $\alpha=0.6$. Here $E_{M S Y}=129.6$ units and $h_{M S Y}=0.16$.

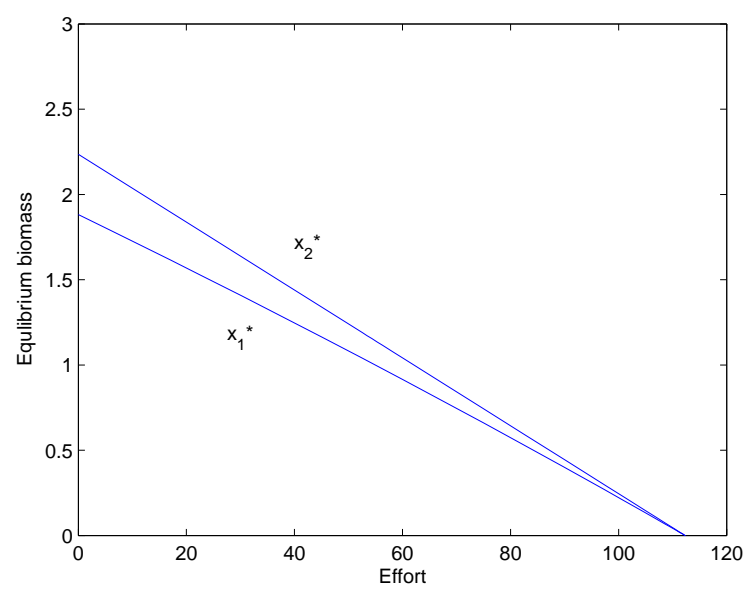

Figure 11a. 


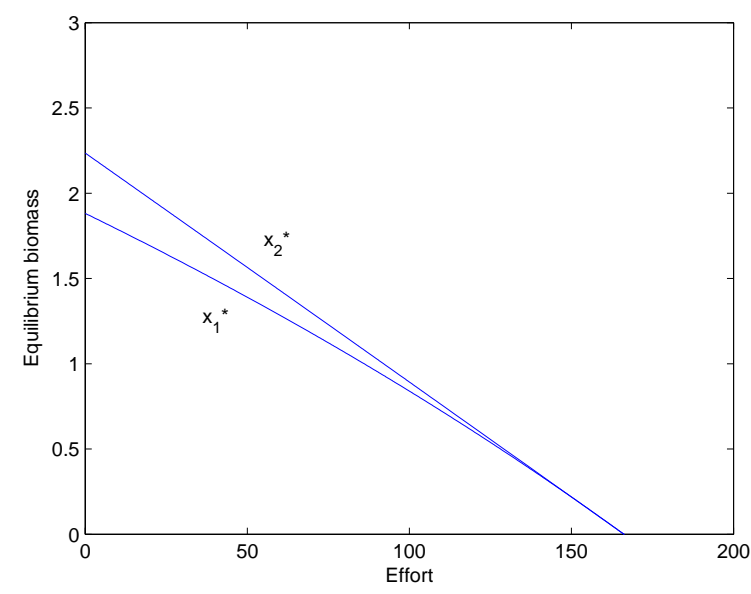

Figure 11b.

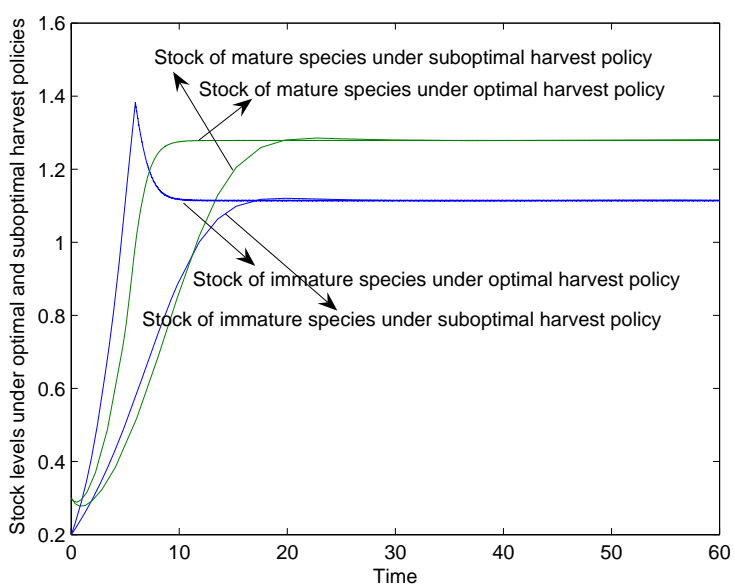

Figure 12. Optimal and suboptimal approach paths for $\alpha=0.4$

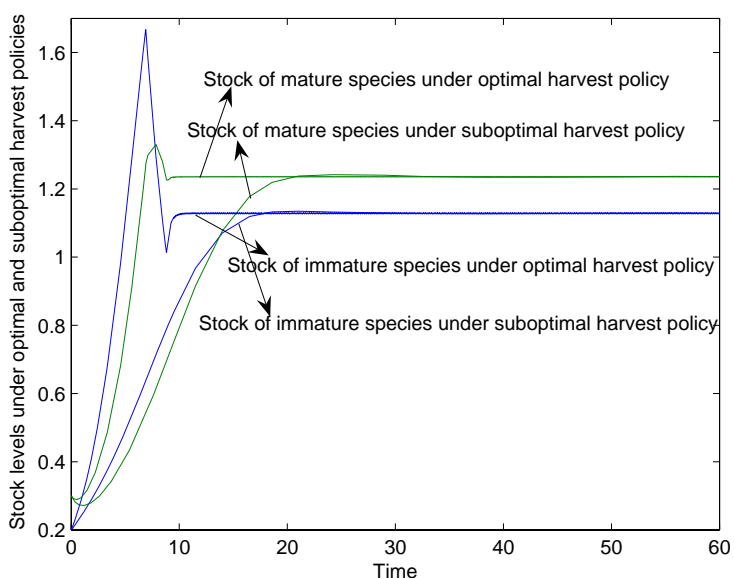

Figure 13. Optimal and suboptimal approach paths for $\alpha=0.6$ 